\title{
Identification of suitable reference genes for quantitative RT-PCR during 3T3-L1 adipocyte differentiation
}

\author{
JUAN ZHANG $^{1 *}$, HONGJU TANG $^{1 *}$, YUQING ZHANG $^{1}$, RUYUAN DENG $^{1}$,

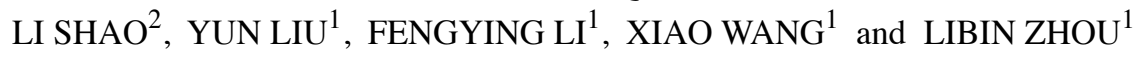 \\ ${ }^{1}$ Shanghai Institute of Endocrine and Metabolic Diseases, Department of Endocrine and Metabolic Diseases, Shanghai \\ Clinical Center for Endocrine and Metabolic Diseases, Ruijin Hospital, Shanghai Jiao Tong University School of Medicine, \\ Shanghai 200025; ${ }^{2}$ Department of Geratology, East Hospital, Shanghai Tongji University, Shanghai 200120, P.R. China
}

Received November 7, 2013; Accepted February 27, 2014

DOI: 10.3892/ijmm.2014.1695

\begin{abstract}
Quantitative reverse transcription PCR (qRT-PCR) is becoming increasingly important in the effort to gain insight into the molecular mechanisms underlying adipogenesis. However, the expression profile of a target gene may be misinterpreted due to the unstable expression of the reference genes under different experimental conditions. Therefore, in this study, we investigated the expression stability of 10 commonly used reference genes during 3T3-L1 adipocyte differentiation. The mRNA expression levels of glyceraldehyde-3-phosphate dehydrogenase (GAPDH) and transferrin receptor (TFRC) significantly increased during the course of 3T3-L1 adipocyte differentiation, which was decreased by berberine, an inhibitor of adipogenesis. Three popular algorithms, GeNorm, NormFinder and BestKeeper, identified 18 ribosomal RNA and hydroxymethylbilane synthase (HMBS) as the most stable
\end{abstract}

Correspondence to: Professor Xiao Wang or Professor Libin Zhou, Shanghai Institute of Endocrine and Metabolic Diseases, Department of Endocrine and Metabolic Diseases, Shanghai Clinical Center for Endocrine and Metabolic Diseases, Ruijin Hospital, Shanghai Jiaotong University School of Medicine, 197 Ruijin 2nd Road, Shanghai 200025, P.R. China

E-mail: wangxiao1976@hotmail.com

E-mail: libin_zhou@yahoo.com

*Contributed equally

Abbreviations: IBMX, 3-isobutyl-1-methylxanthine; qRT-PCR, quantitative reverse transcription-polymerase chain reaction; GAPDH, glyceraldehyde-3-phosphate dehydrogenase; actin, $\beta$-actin; $18 \mathrm{~S}$, 18 ribosomal RNA; HMBS, hydroxymethylbilane synthase; TFRC, transferrin receptor; PPAR $\gamma 2$, peroxisome proliferator-activated receptor $\gamma 2$; C/EBP, CCAAT/enhanced binding protein; BSA, bovine serum albumin; FBS, fetal bovine serum; PBS, phosphate-buffered saline; PIPA, cyclophilin A; 36-B4, ribosomal protein, large, P0; HPRT, hypoxanthine-guanine phosphoribosyltransferase; B2M, beta-2microglobulin

Key words: reference gene, quantitative reverse transcriptionpolymerase chain reaction, adipocyte differentiation, berberine, glyceraldehyde-3-phosphate dehydrogenase reference genes, while GAPDH and TFRC were the least stable ones. Peptidylprolyl isomerase A [PIPA (cyclophilin A)], ribosomal protein, large, P0 (36-B4), beta-2-microglobulin (B2M), $\alpha 1$-tubulin, hypoxanthine-guanine phosphoribosyltransferase (HPRT) and $\beta$-actin showed relatively stable expression levels. The choice of reference genes with various expression stabilities exerted a profound influence on the expression profiles of 2 target genes, peroxisome proliferator-activated receptor (PPAR) $\gamma 2$ and C/EBP $\alpha$. In addition, western blot analysis revealed that the increased protein expression of GAPDH was markedly inhibited by berberine during adipocyte differentiation. This study highlights the importance of selecting suitable reference genes for qRT-PCR studies of gene expression during the process of adipogenesis.

\section{Introduction}

Obesity has become a widespread issue in modern society. When energy input exceeds energy expenditure, adipose tissue mass increases by adipocyte hyperplasia and hypertrophy (1). In order to manage the health concerns associated with obesity, it is necessary to understand its development and regulation. At the cellular level, obesity is considered a hypertrophic disease resulting from an increase in adipogenesis (2). The 3T3-L1 cell line derived from 3T3 Swiss mouse embryo is one of the most well-characterized and reliable models for studying adipogenesis. Confluent 3T3-L1 preadipocytes differentiate upon exposure to adipogenic inducers, such as insulin, 3-isobutyl1-methylxanthine (IBMX) and dexamethasone. These inducers activate the dramatic changes in cell morphology, cytoskeletal components and the level and type of extracellular matrix components. The acquisition of the adipocyte phenotype is characterized by chronological changes in the expression of numerous genes (3). It is crucial to identify the expression profiles of specific genes during the process of adipogenesis, which would provide important insight into the molecular mechanisms underlying adipogenesis.

Quantitative reverse transcription-polymerase chain reaction (qRT-PCR) is a powerful and efficient means of rapidly comparing gene expression patterns between different developmental stages and experimental conditions (4). To ensure reproducible and accurate quantitative expression measures, it 
is necessary to normalize the expression levels of target genes using suitable reference genes. An ideal reference gene should show similar mRNA levels at different stages of development of an organism or in different tissues, and should not vary in abundance in response to environmental factors or bioassay treatments. However, there is no universal reference gene with a constant expression in all tissues and experimental conditions (5). Increasing evidence demonstrates that the expression levels of the most commonly used internal reference genes, including glyceraldehyde-3-phosphate dehydrogenase (GAPDH), $\beta$-actin (actin) and 18 ribosomal RNA (18S) vary markedly under different experimental conditions (6-9). Normalization using unsuitable reference genes will lead to erroneous results (6-9). Therefore, the selection of appropriate reference genes is critical for the interpretation and accuracy of expression data.

In this study, 3 popular algorithms, GeNorm (10), NormFinder (11) and BestKeeper (12) were used to evaluate the expression stability of 10 commonly used reference genes throughout 3T3-L1 adipocyte differentiation. We identified $18 \mathrm{~S}$ and hydroxymethylbilane synthase (HMBS) as the most stable internal reference genes, while GAPDH and transferrin receptor (TFRC) were the least stable ones for adipocyte differentiation studies. We also analyzed the influence of various reference genes on the expression profiles of target genes, such as 2 key transcript factors for adipocyte differentiation, peroxisome proliferator-activated receptor (PPAR) $\gamma 2$ and CCAAT/ enhanced binding protein $(\mathrm{C} / \mathrm{EBP}) \alpha(2,3)$. The use of GAPDH and TFRC as reference genes significantly underestimated the changes in the expression levels of these genes.

\section{Materials and methods}

Materials. Dulbecco's modified Eagle's medium (DMEM) and other culture reagents were obtained from Gibco Life Technologies (Grand Island, NY, USA). The cell culture plates were purchased from Nalge Nunc International (Roskilde, Denmark). Human insulin (HumulinR) was obtained from Eli Lilly S.A.S. (Fegersheim, France). Bovine serum albumin (BSA), IBMX and dexamethasone were purchased from Sigma (St. Louis, MO, USA). Anti-actin, anti-GAPDH, anti- $\alpha 1$-tubulin (tubulin), anti-mouse IgG and anti-rabbit IgG conjugated with horseradish peroxidase were obtained from Cell Signaling Technology (Beverly, MA, USA). Murine-derived 3T3-L1 preadipocytes were purchased from the American Type Culture Collection (ATCC; Rockville, MD, USA). Berberine was obtained from the National Institute for the Control of Pharmaceutical and Biological Products (Beijing, China).

Cell culture and differentiation. 3T3-L1 preadipocytes were grown and passaged in DMEM containing $25 \mathrm{mM}$ glucose plus $10 \%$ fetal bovine serum (FBS). For adipocyte differentiation, 2-day post-confluent cells were placed in $10 \%$ FBS-DMEM with $250 \mathrm{nM}$ dexamethasone, $0.5 \mathrm{mM}$ IBMX and $1 \mu \mathrm{g} / \mathrm{ml}$ insulin. After 2 days, the medium was changed to $10 \%$ FBS-DMEM containing $1 \mu \mathrm{g} / \mathrm{ml}$ insulin alone for 2 additional days and was replaced with $10 \%$ FBS-DMEM. Thereafter, the medium was changed every 2 days.

Oil Red $O$ staining. 3T3-L1 preadipocytes induced to differentiate for various days were washed with phosphate-buffered saline (PBS), fixed with $4 \%$ paraformaldehyde in $0.1 \mathrm{M}$ phosphate buffer, $\mathrm{pH} 7.4$ for $15 \mathrm{~min}$ at room temperature, and washed 3 times with deionized water. A mixture of Oil Red O (0.6\% Oil Red O dye in isopropanol) and water at a 6:4 ratio was layered on the cells for $10 \mathrm{~min}$, followed by hematoxylin counterstaining.

$q R T-P C R$. Total RNA was isolated using TRIzol reagent (Invitrogen, Carlsbad, CA, USA) and reverse transcribed from random primers (Promega, Madison, WI, USA) according to the manufacturer's instructions. Real-time PCR was performed on a Roche LightCycler 480 system using SYBR Premix $\mathrm{Ex} \mathrm{Taq}^{\mathrm{TM}}$ (Takara, Otsu, Japan) in a final volume of $20 \mu \mathrm{l}$. The conditions for real-time PCR were as follows: denaturation at $95^{\circ} \mathrm{C}$ for $10 \mathrm{sec}, 40$ cycles at $95^{\circ} \mathrm{C}$ for $5 \mathrm{sec}$, and $60^{\circ} \mathrm{C}$ for 31 sec. A melting curve was built in the temperature range of $60-95^{\circ} \mathrm{C}$ at the end of amplification. The primer sequences used for real-time PCR are presented in Table I. All primers were synthesized by Shanghai Sangon Biological Engineering Technology \& Services Co., Ltd. (Shanghai, China).

Western blot analysis. Cells in 6-well plates were washed twice with ice-cold PBS and placed immediately in lysis buffer containing $1 \mathrm{mM}$ phenylmethylsulfonyl fluoride (PMSF), protease inhibitor cocktail I (Calbiochem/EMD Miliipore, Billerica, MA, USA) and phosphatase inhibitor cocktail V (Merck, Darmstadt, Germany). Lysates were gently mixed for $10 \mathrm{~min}$ at $4^{\circ} \mathrm{C}$ and then centrifuged at $13,000 \times \mathrm{g}$ for $15 \mathrm{~min}$ at $4^{\circ} \mathrm{C}$. The protein concentration of the extracts was determined according to the method of Bradford, using BSA as the standard. Samples were separated by SDS-PAGE on $8 \%$ polyacrylamide gels and transferred onto PVDF-Plus membranes (Bio-Rad, Hercules, CA, USA). Primary antibodies were detected with donkey anti-rabbit at 1:2,000 for $1 \mathrm{~h}$ at room temperature. The blotted membrane was developed with ECL Advance (Cell Signaling Technology, Boston, MA, USA) and imaged with a LAS-4000 Super CCD Remote Control Science Imaging System (Fuji, Tokyo, Japan).

Software determination of appropriate reference genes and statistical analysis. For stability comparisons of candidate reference genes, 3 validation software programs, GeNorm, NormFinder and BestKeeper, were used according to their original publications (10-12). Data are presented as the means \pm SEM. Comparisons were performed using ANOVA for multiple groups or the Student's t-test for 2 groups. A value of $\mathrm{P}<0.05$ was considered to indicate a statistically significant difference.

\section{Results}

Quality control for the accuracy of gene expression by $q R T-P C R$. For obtaining accurate and reliable results of gene expression, attention should be paid to well-differentiated adipocytes, equal sample size, intact RNA and efficient primers apart from reference genes $(5,13)$. Whether preadipocytes converge into mature adipocytes is important for research. As shown in Fig. 1, 3T3-L1 preadipocytes exhibited a similar morphology to fibroblasts. The induction of differentiation for 4 days triggered deep phenotypical changes of preadipo- 


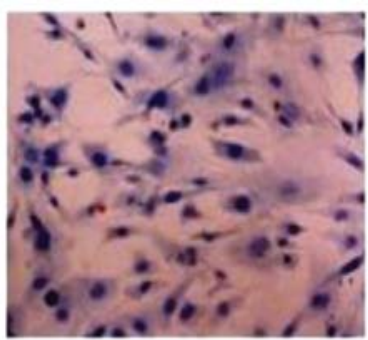

Preadipocytes

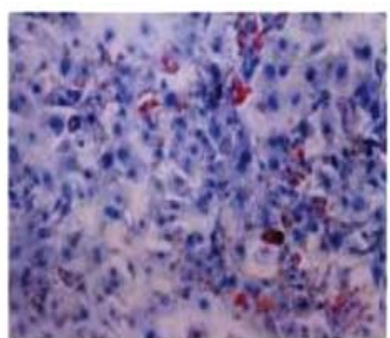

4 days post-MDI

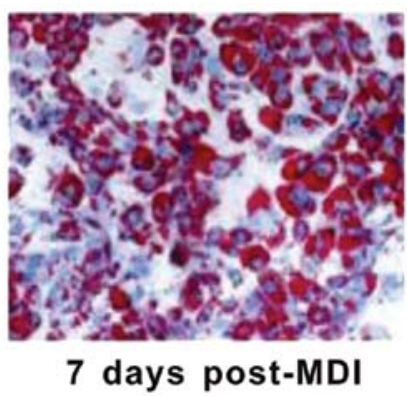

7 days post-MDI

Figure 1. 3T3-L1 adipocyte differentiation. 3T3-L1 preadipocytes were induced to differentiate into mature adipocytes with MDI (3-isobutyl-1-methylxanthine, dexamethasone and insulin). 3T3-L1 cells on various days of differentiation were stained by Oil Red O.

A

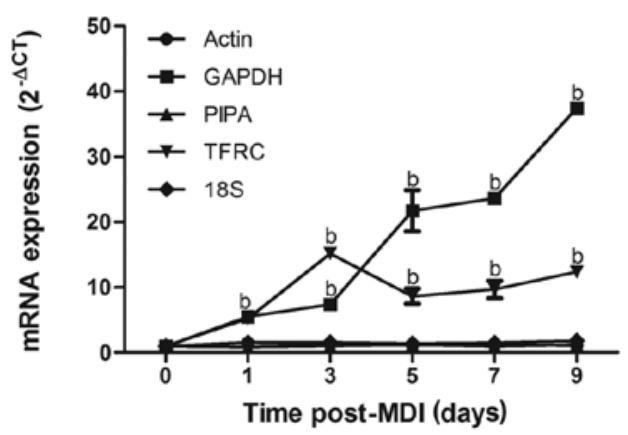

B

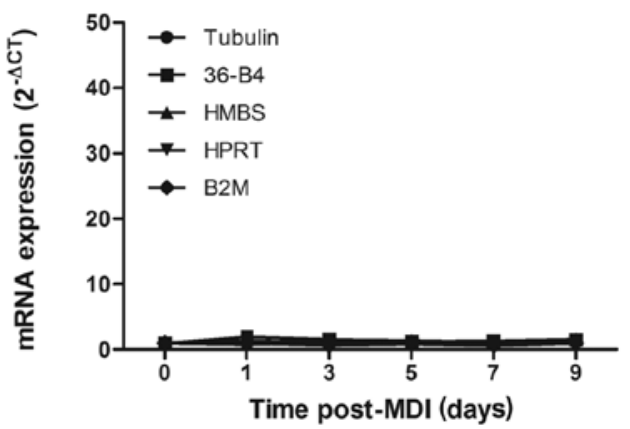

Figure 2. (A and B) Relative expression levels of candidate reference genes without normalization. Total RNA was isolated from 0 to 9 days after the 3T3-L1 preadipocytes were treated with MDI (3-isobutyl-1-methylxanthine, dexamethasone and insulin) and assessed for reference gene expression by qRT-PCR. Relative quantification analysis of gene expression data was conducted according to the $2^{-\Delta \mathrm{CT}}$ method. Data are expressed as the relative fold to day 0 . Significance was established at ${ }^{\mathrm{b}} \mathrm{P}<0.01$ compared with day 0 .

cytes that became spherical, with small lipid droplets in the cytoplasm, as shown by Oil Red O staining. Seven days after induction, $>90 \%$ of the cells showed the phenotype of mature adipocytes, with a large number of lipid droplets accumulated in the cytoplasm. To reduce inter-sample variation, total RNA of 3T3-L1 adipocytes at different time points after the induction of differentiation was isolated and reverse transcribed at simultaneously. The mean A260/280 ratio of the RNA samples was $1.98 \pm 0.03$ and reflected pure and protein-free RNA. The integrity of RNA samples was characterized by an $28 \mathrm{~S} / 18 \mathrm{~S}$ ratio of $>2$ on a $1 \%$ agarose gel. Real-time PCR was run in duplicate and all samples at different time points were analyzed in the same run in order to exclude between-run variations. A melting curve was constructed for each primer pair to confirm product specificity.

Non-normalized expression levels of candidate reference genes during 3T3-L1 adipocyte differentiation. Another essential strategy suggested for normalizing qRT-PCR data is to use a suitable internal reference gene whose expression should not change with treatment or under different experimental conditions (5). It is important to distinguish technical variability from true biological changes in gene expression. According to the guidelines described in the study by Gorzelniak (14), the difference in threshold cycle number $(\triangle \mathrm{CT})$ values before and after induction $(< \pm 0.5)$ is considered a fluctuation in gene expression that is largely due to technical variance, while the $\Delta \mathrm{CT}$ values $(> \pm 1.0)$ are strongly suggestive of biological variability resulting from treatment or experimental conditions. Among the 10 reference genes (Table I), the relative expression levels of actin, peptidylprolyl isomerase A [PIPA (cyclophilin A)], 18S, tubulin, ribosomal protein, large, P0 (36-B4), HMBS, hypoxanthine-guanine phosphoribosyltransferase (HPRT) and beta-2-microglobulin (B2M) fluctuated within the range $\Delta \mathrm{CT}< \pm 0.5$ during the course of 3T3-L1 adipocyte differentiation (Fig. 2A and B), precluding these commonly used reference genes for suitability under this experimental condition. On the contrary, GAPDH and TFRC were disqualified as suitable reference genes, with $\triangle \mathrm{CT}$ values $> \pm 1.0$ clearly indicative of biological variability. The GAPDH mRNA level increased by 6.2-, 7.5-, 22.1-, 24.1- and 17.5-fold on day $1,3,5,7$ and 9, respectively after the induction of differentiation $(\mathrm{P}<0.01)$; the mRNA level of TFRC increased by 5.2-, 15.2-, 8.7-, 9.7- and 12.4-fold on day 1, 3, 5, 7 and 9, respectively $(\mathrm{P}<0.01$, Fig. $2 \mathrm{~A})$.

Statistical validation of appropriate reference genes by GeNorm, NormFinder and BestKeeper. To identify the most suitable set of genes for normalization during 3T3-L1 adipocyte differentiation, the expression stability of the 10 candidate reference genes was analyzed using the GeNorm, NormFinder and BestKeeper programs. The GeNorm algorithm determines expression stability (M) through a pair-wise comparison of one candidate reference gene and all other candidate genes, independent of the level of gene expression for each sample $(10,15)$. The genes with the lowest M-values will be considered to have 


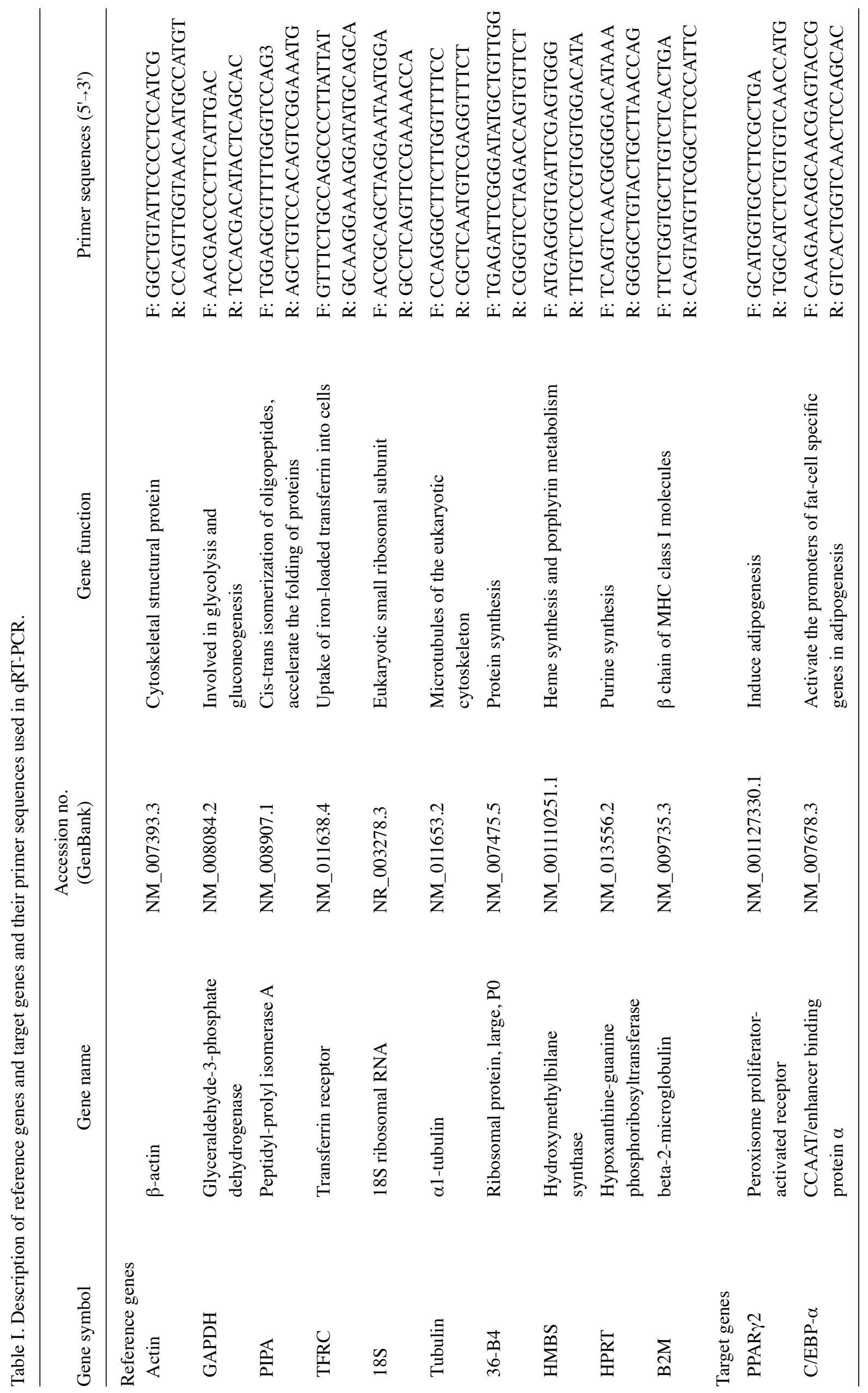



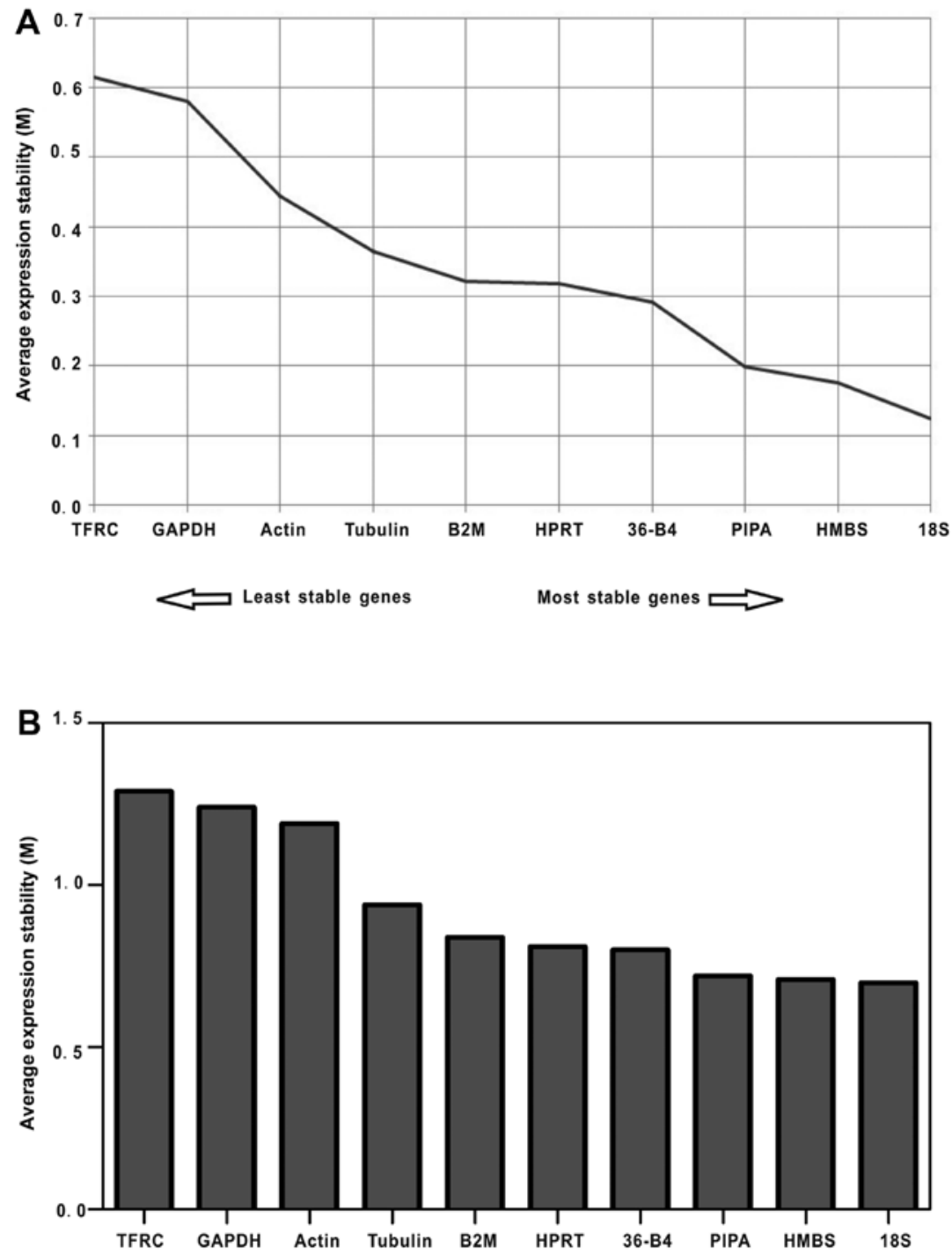

Figure 3. Expression stability analysis of reference genes by GeNorm and NormFinder. Average expression stability values (M) of 10 reference genes were calculated by (A) GeNorm and (B) NormFinder. Reference genes were graphed to illustrate the average gene expression stability on the y-axis and its associated ranking from least to most stable expression on the x-axis. Lower M-values of average expression stability indicate more stable expression.

the most stable expression during 3T3-L1 adipocyte differentiation (0-9 days). As a result, the ranking of the M-values of the examined reference genes was as follows: $18 \mathrm{~S}>\mathrm{HMBS}>$ PIPA $>36-$ B4 $>$ HPRT $>$ B2M $>$ tubulin $>$ actin $>$ GAPDH $>$ TFRC (Fig. 3A).

NormFinder was designed to calculate stability by using the combined estimation of intra- and intergroup expression variations of the analyzed genes (11). Based on the calculated stability values of the 10 reference genes shown in Fig. 3B, the NormFinder program validated the findings of the GeNorm algorithm, in which the most unstable reference genes were TFRC and GAPDH, and the most stable one was 18S followed by HMBS.

BestKeeper calculates the percentage coefficient of variation (CV) and standard deviation (SD) using the crossing point (CP) value of each candidate gene $(12,16)$. As shown in Table II, GAPDH and TFRC remained the most unstable reference genes, whereas HMBS and PIPA were ranked the most stable reference genes, a finding that was different from the other 2 software results.
Taken together, the software analysis results indicated that GAPDH and TFRC ranked as the least stable reference genes, while 18S and HMBS ranked as the most stable ones (Table III). Therefore, GAPDH and TFRC may not be a suitable choice for use as reference genes, whereas 18S and HMBS may serve well as reference genes during 3T3-L1 adipocyte differentiation.

Expression levels of target gene influenced by the selection of normalized genes. To illustrate the impact of reference gene selection on the determination of target gene expression levels, the expression profiles of 2 key transcription factors in adipocyte differentiation, $\operatorname{PPAR} \gamma 2$ and $\mathrm{C} / \operatorname{EBP} \alpha(2,17)$, were individually normalized to 4 different reference genes with varying degrees of suitability. We determined the nonnormalized mRNA expression profile of 2 target genes in the same samples used in the determination of the reference gene expression profile. The expression of PPAR $\gamma 2$ was gradually increased with the differentiation of 3T3-L1 preadipocytes, reaching the peak with a 676 -fold increase 7 days after induc- 
Table II. Detailed expression stability analysis of candidate reference genes by BestKeeper software.

\begin{tabular}{|c|c|c|c|c|c|c|c|c|c|c|}
\hline Gene & Actin & GAPDH & PIPA & TFRC & $18 \mathrm{~S}$ & Tubulin & $36-\mathrm{B} 4$ & HMBS & HPRT & $\mathrm{B} 2 \mathrm{M}$ \\
\hline $\mathrm{n}$ & 36 & 36 & 36 & 36 & 36 & 36 & 36 & 36 & 36 & 36 \\
\hline geo Mean (CP) & 20.57 & 19.18 & 22.27 & 26.57 & 12.79 & 20.97 & 22.13 & 27.06 & 23.68 & 19.25 \\
\hline ar Mean (CP) & 20.59 & 19.23 & 22.27 & 26.60 & 12.80 & 20.97 & 22.14 & 27.06 & 23.70 & 19.25 \\
\hline $\operatorname{Min}(\mathrm{CP})$ & 19.38 & 17.07 & 21.55 & 24.73 & 12.05 & 20.06 & 20.95 & 26.22 & 22.54 & 18.18 \\
\hline $\operatorname{Max}(\mathrm{CP})$ & 21.72 & 21.47 & 22.92 & 28.57 & 13.57 & 21.88 & 23.59 & 27.62 & 25.25 & 20.24 \\
\hline Std dev. $( \pm \mathrm{CP})$ & 0.59 & 1.18 & 0.31 & 1.03 & 0.49 & 0.37 & 0.56 & 0.27 & 0.62 & 0.32 \\
\hline $\mathrm{CV}(\% \mathrm{CP})$ & 2.88 & 6.13 & 1.38 & 3.86 & 3.79 & 1.77 & 2.54 & 0.99 & 2.60 & 1.66 \\
\hline Min (x-fold) & -2.29 & -4.32 & -1.64 & -3.59 & -1.68 & -1.88 & -2.27 & -1.79 & -2.22 & -2.10 \\
\hline Max (x-fold) & 2.21 & 4.87 & 1.57 & 3.99 & 1.71 & 1.87 & 2.76 & 1.47 & 2.96 & 1.98 \\
\hline Std dev. ( $\pm \mathrm{X}$-fold $)$ & 1.51 & 2.26 & 1.24 & 2.04 & 1.40 & 1.29 & 1.48 & 1.20 & 1.53 & 1.25 \\
\hline
\end{tabular}

Descriptive statistics of 10 reference genes are shown based on their crossing point $(\mathrm{CP})$ values. n, number of samples; geo Mean $(\mathrm{CP})$, geometric mean value of the $\mathrm{CP}$; ar Mean $(\mathrm{CP})$, average mean value of the $\mathrm{CP}$; Min $(\mathrm{CP})$ and Max $(\mathrm{CP})$, extreme values of $\mathrm{CP}$; Std dev. $( \pm \mathrm{CP})$, standard deviation of the CP; CV (\% CP), coefficient of variance expressed as a percentage on the CP level; Min (x-fold) and Max (x-fold), extreme values of expression levels expressed as absolute $\mathrm{x}$-fold over or under regulation coefficient; Std dev. ( $\pm \mathrm{x}$-old), standard deviation of the absolute regulation coefficients.

A

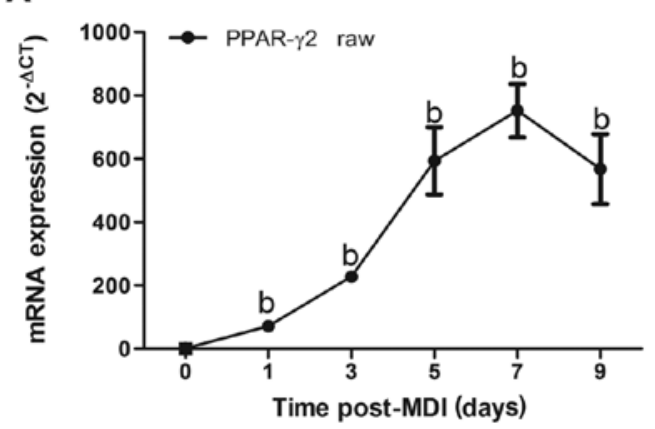

C

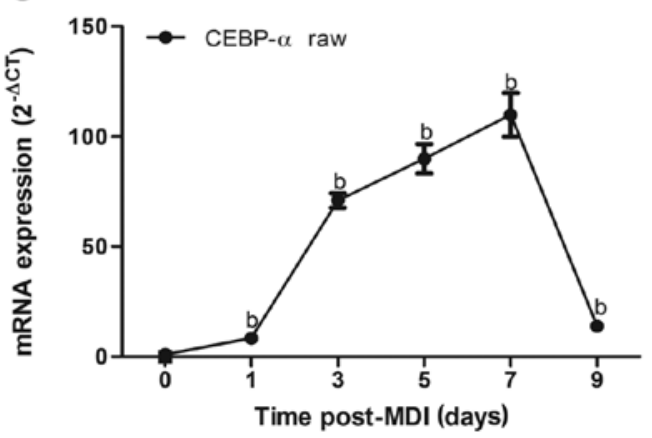

B

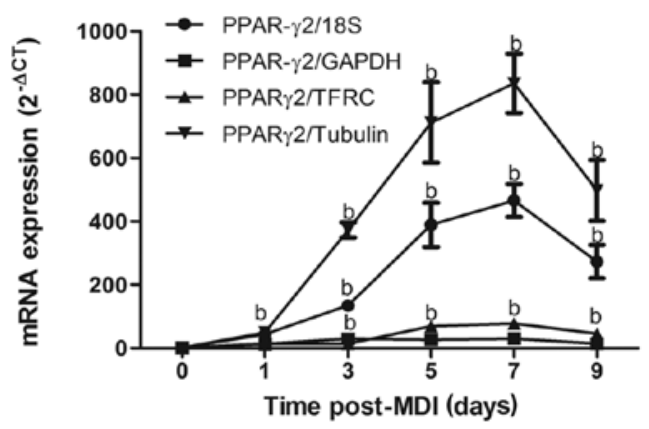

D

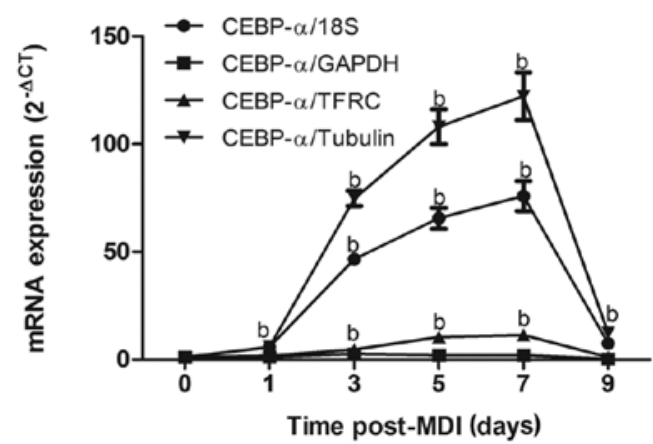

Figure 4. Relative expression levels of different target genes without and with normalization. Total RNA was isolated from 0 to 9 days after the 3T3-L1 preadipocytes were treated with MDI (3-isobutyl-1-methylxanthine, dexamethasone and insulin) and assessed for reference gene expression by qRT-PCR. Changes in PPAR $\gamma 2$ gene expression (A) without and with (B) normalization to 18S, tubulin, GAPDH, or TFRC are shown. (C) Raw gene expression and (D) normalized expression of $\mathrm{C} / \mathrm{EBP} \alpha$. Significance was established at ${ }^{\mathrm{b}} \mathrm{P}<0.01$ compared with day 0 .

tion (Fig. 4A). The expression profile of PPAR $\gamma 2$ normalized to $18 \mathrm{~S}$ or tubulin was consistent with its raw expression, which gradually increased 1 day after induction and reached its peak 7 days later. However, the magnitude of PPAR $\gamma 2$ expression was markedly attenuated when normalized to GAPHD or TFRC, reaching only a 28 - or 70 -fold increase, respectively as compared to a 466-fold or 551-fold increase when normalized to $18 \mathrm{~S}$ or tubulin, respectively 7 days after induction (Fig. 4B). Similarly, the magnitude of $\mathrm{C} / \mathrm{EBP} \alpha$ expression when normalized to GAPDH was sharply decreased compared with its raw expression (Fig. 4C and D). Collectively, these data demonstrate that the selection of reference genes exerts a 
A
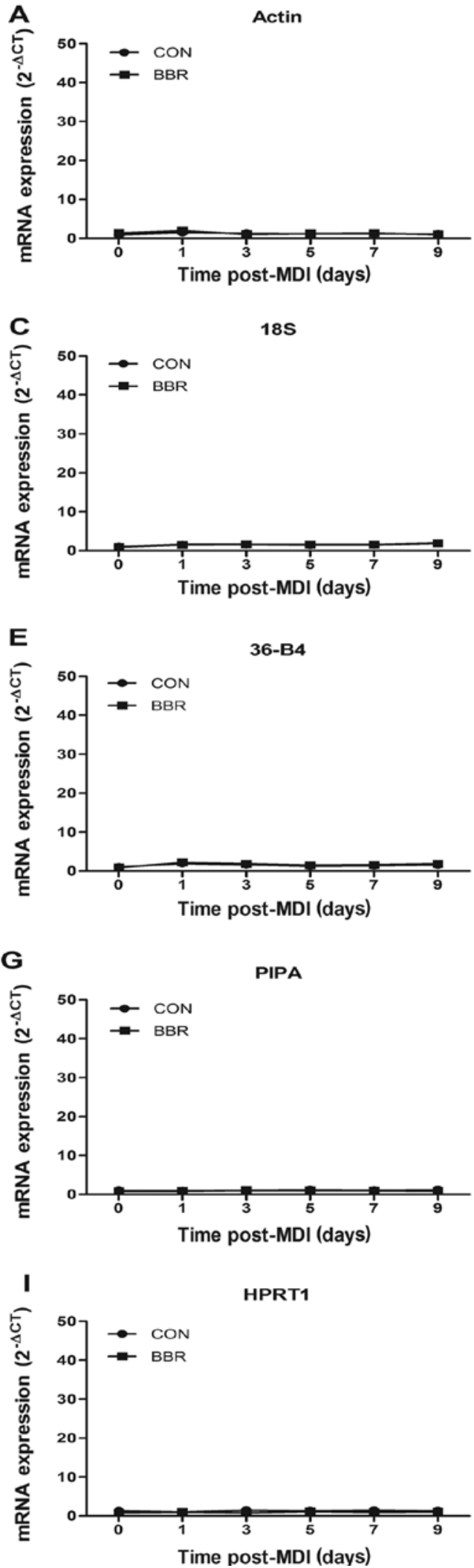

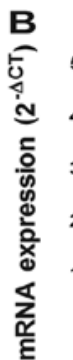

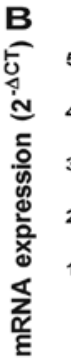
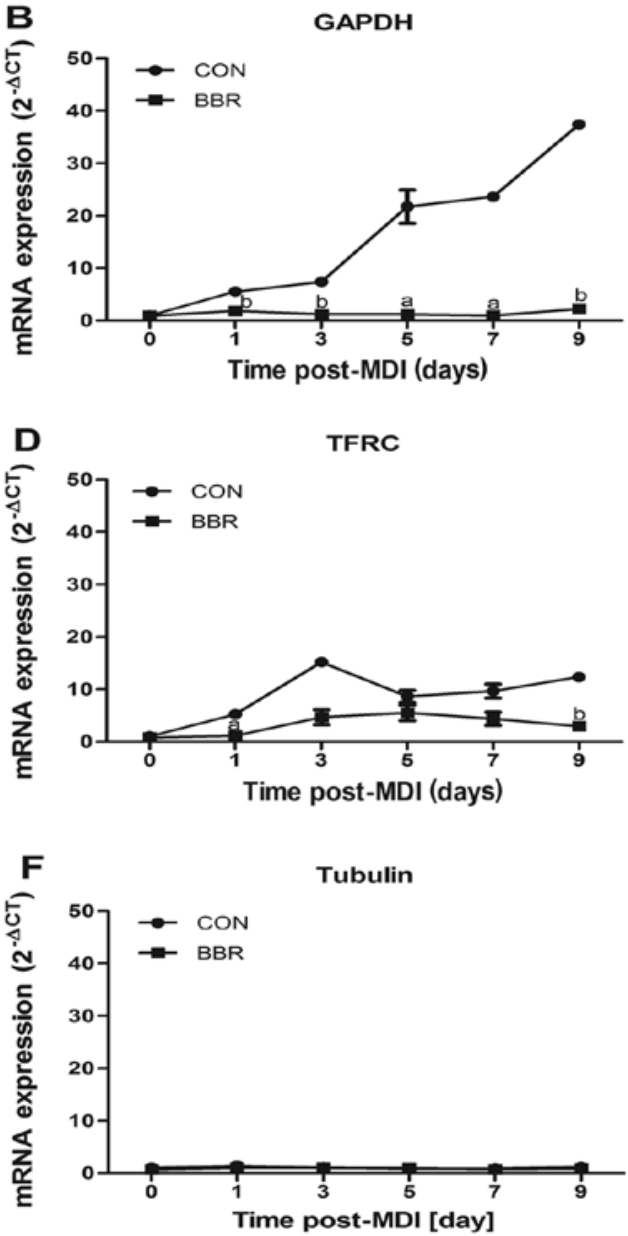

H
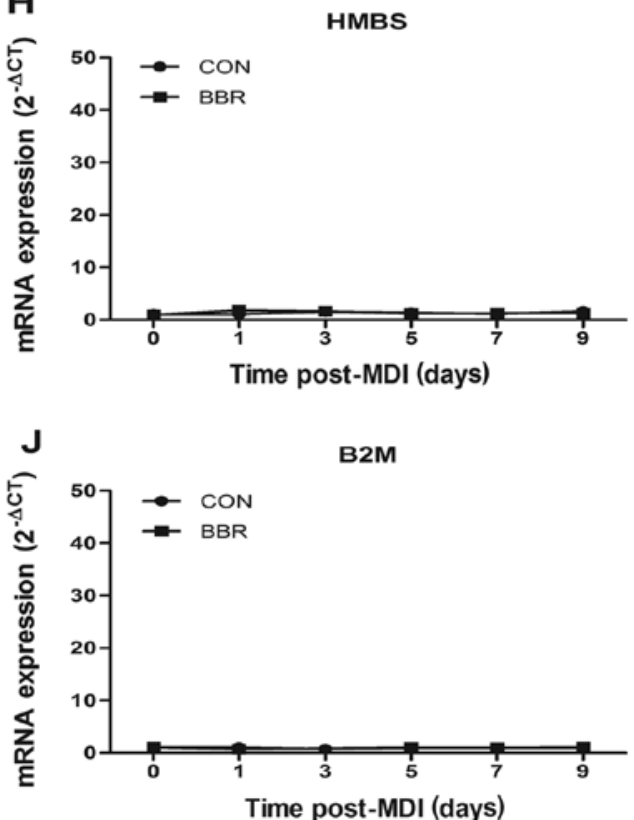

Figure 5. Effects of berberine on mRNA expression levels of candidate reference genes. Total RNA was isolated from the differentiated 3T3-L1 cells (0-9 days) in the presence or absence of $5 \mu \mathrm{M}$ berberine (BBR). Relative quantification analysis of (A) actin, (B) GAPDH, (C) 18S, (D) TFRC, (E) 36-B4, (F) tubulin, (G) PIPA, (H) HMBS, (I) HPRT, (J) B2M gene expression levels was conducted by the $2^{-\triangle C T}$ method. Data are expressed as the relative fold to day 0 . Significance was established at ${ }^{\mathrm{a}} \mathrm{P}<0.05,{ }^{\mathrm{b}} \mathrm{P}<0.01$ compared with day 0 .

profound impact on the experimental outcome, illustrating the critical need to validate each reference gene in a cell-type and condition-specific manner.
Effects of berberine on the expression levels of reference genes. Berberine, one of the major constituents of the Chinese herb, Rhizoma coptidis, has been reported to improve insulin 


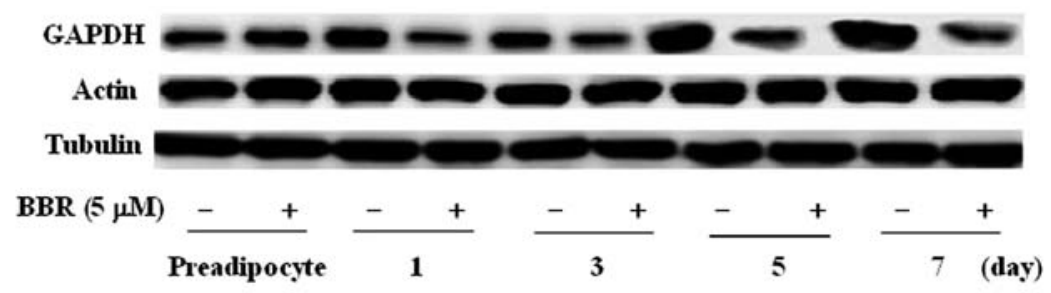

Figure 6. The protein expression levels of 3 commonly used reference genes. 3T3-L1 preadipocytes were induced to differentiate into mature adipocytes with insulin, 3-isobutyl-1-methylxanthine and dexamethasone. Berberine (BBR) (5 $\mu \mathrm{M})$ was added from the beginning of induction. The cells at various stages of differentiation were collected for analyzing GAPDH, actin and tubulin protein expression levels by western blot analysis.

Table III. Ranking of reference gene stability during 3T3-L1 adipocyte differentiation.

\begin{tabular}{llll}
\hline Ranking & BestKeeper & GeNorm & NormFinder \\
\hline 1 & HMBS & 18S & 18S \\
2 & PIPA & HMBS & HMBS \\
3 & B2M & PIPA & PIPA \\
4 & Tubulin & $36-$ B 4 & $36-B 4$ \\
5 & $18 S$ & HPRT & B2M \\
6 & $36-B 4$ & B2M & Tubulin \\
7 & Actin & Tubulin & HPRT \\
8 & HPRT & Actin & Actin \\
9 & TFRC & GAPDH & TFRC \\
10 & GAPDH & TFRC & GAPDH \\
\hline
\end{tabular}

Reference gene stability is ranked in order of decreasing expression stability.

resistance and reduce hyperglycemia $(18,19)$. Our previous study indicated that berberine significantly inhibited 3T3-L1 adipocyte differentiation (20), which has also been confirmed by other studies $(21,22)$. To further analyze the expression stability of the reference genes, $5 \mu \mathrm{M}$ berberine were added to the cell culture during the differentiation of 3T3-L1 adipocytes. The results revealed that GAPDH mRNA expression was significantly reduced by berberine at all stages of adipocyte differentiation $(\mathrm{P}<0.05$ or $\mathrm{P}<0.01)$, reduced by 68 and $66 \%$ at 5 and 7 days, respectively. As expected, there were no significant changes in the expression levels of the other 8 reference gene in the presence of berberine apart from TFRC (Fig. 5).

In addition, we further detected the protein expression levels of 3 commonly used reference genes. Western blot analysis revealed that GAPDH protein expression markedly increased at 5 and 7 days after induction, while there were no obvious changes in actin and tubulin protein expression throughout adipocyte differentiation. GAPDH protein expression levels were inhibited by berberine, but not those of actin and tubulin (Fig. 6). Consequently, GAPDH is not appropriate as a reference gene for western blot analysis during 3T3-L1 adipocyte differentiation.

\section{Discussion}

qRT-PCR has become one of the most popular techniques for quantifying mRNA levels, particularly for low-abundance genes. For the accurate and reliable analysis of target gene expression, the normalization of qRT-PCR data with suitable internal reference genes is required (5). Normalization is essential to correct the non-specific variations arising from the difference in the amount of template used and its quality, which can affect the efficiency of the qRT-PCR reactions (11). The strategy is based on the assumption that reference genes are stably expressed. However, there is mounting evidence suggesting that the expression of internal reference genes may vary significantly under different experimental conditions, opening the possibility that erroneous information could be generated if data normalization is based on genes that themselves are regulated $(9,23,24)$. Reference genes are essential endogenous regulatory genes that are involved in various processes in the cell, such as metabolism, cell structure, gene transcription and homeostasis (25). Adipocyte differentiation is a process where cells undergo marked morphological changes, accompanied by substantial biochemical changes, such as cell cycle exit and changes in biochemical processes, metabolism and cytoskeletal components $(3,26)$. Significant modifications in gene expression therefore underlie these vast arrays of protein and cellular changes. Therefore, in this study, we evaluated 10 commonly used candidate reference genes for normalizing qRT-PCR gene expression data during the course of 3T3-L1 adipocyte differentiation.

Firstly, we evaluated the differentiation status of 3T3-L1 adipocytes using Oil Red O staining. The results revealed that 3T3-L1 adipocytes were filled with lipid 7 days after the induction of differentiation, along with a significant increase in the PPAR $\gamma 2$ mRNA level. Therefore, these cells were considered to be fully differentiated. Secondly, the samples of various groups were simultaneously extracted, reversed and amplified to reduce intersample variability. We directly compared the CT values of all candidate reference genes in 3T3-L1 adipocytes differentiated for different periods of time (different number of days). As $\Delta \mathrm{CT}$ values are exponents, all validation data were converted to 'fold changes' using the $2^{-\triangle C T}$ method for raw data. Accordingly, a $\Delta \mathrm{CT}$ value of -0.5 and +0.5 is equivalent to 0.7 - and 1.4 -fold changes in relative gene expression, respectively. Using the criterion of $\Delta \mathrm{CT} \leq \pm 0.5$ as a delimiter of reference gene suitability, we identified 8 reference genes (actin, PIPA, 18S, tubulin, 36-b4, HMBS, HPRT and B2M) suitable for use in target gene normalization. However, GAPDH and TFRC mRNA expression levels fluctuated with $(\Delta \mathrm{CT}>1.0)$ after 3T3-L1 adipocyte differentiation, showing dramatic increases as compared to before induction. Thus, the 2 genes were inappropriate as endogenous reference genes under this experimental condition. 
As a simple comparison of $\mathrm{CT}$ values revealed an 'overall expression variation' for the candidate reference genes, we further evaluated the expression stability of the 10 candidate reference genes using 3 software programs. Despite the differences we found in the reference gene ranking when using different statistical approaches, there was a general agreement among the methods for the determination of the most stable and unstable genes. The GeNorm and NormFinder identified $18 \mathrm{~S}$ and HMBS as the most stable genes, while BestKeeper recommended HMBS and PIPA as the most stable ones. The 3 software programs all identified GAPDH and TFRC as the least stable genes, which confirmed the simple comparison of the CT value according to the guideline described in the study by Gorzelniak (14). Previous studies have revealed the expression stability of $18 \mathrm{~S}$ during 3T3-L1 adipocyte differentiation and in human adipose tissue $(7,27)$, as well as under a number of experimental conditions $(8,28)$. Therefore, $18 \mathrm{~S}$, as opposed to HMBS was recommended for normalizing target gene expression in this study.

GAPDH is a classic glycolytic enzyme and has been traditionally considered as a reference gene, the levels of which are considered remain stable under different manipulations. Two previous studies have demonstrated that GAPDH is a stably expressed gene in human adipose cells across a wide range of experimental settings (14) and in the epicardial adipose tissues of lean, overweight and obese patients undergoing coronary artery bypass grafting (29). Even in studies involving adipocyte differentiation, GAPDH was still seleced as an endogenous reference gene $(30,31)$. However, substantial evidence has revealed that the GAPDH gene expression level varies considerably in adipose tissue $(27,32)$ and in other tissues or cultured cells under different situations $(9,33,34)$. As demonstrated in differentiating adipocytes $(6,7)$ and in adipose tissue derived from genetically obese rats $(35,36)$, the upregulation of GAPDH parallels the acquisition of the full lipogenic phenotype. Moreover, GAPDH mRNA expression is stimulated by multiple factors, such as insulin, AMP analogs, T3 and norepinephrine involved in adipocyte differentiation $(6,37)$. Therefore, GAPDH is considered an important adipogenic marker $(38,39)$. In the present study, GAPDH mRNA and protein levels were gradually increased with the maturity of differentiated 3T3-L1 adipocytes, particularly at the later stages of differentiation, which was abrogated by berberine, an inhibitor of adipogenesis. Thus, GAPDH is not recommended as an endogenous control gene during adipocyte differentiation for qRT-PCR as well as western blot analysis.

Insulin elicits a redistribution in TFRC expression in 3T3-L1 adipocytes through an increase in the rate constant for receptor externalization (40). TFRC mRNA expression has been shown to be upregulated in omental and subcutaneous adipose tissue of obese patients (27). Gabrielsen et al observed a $40 \%$ decrease in TFRC mRNA expression in adipocytes from mice fed a high-iron diet compared with that in mice fed normal chow (41). In the current study, the increased TFRC mRNA expression throughout 3T3-L1 adipocyte differentiation was reduced by berberine, suggesting that TFRC is an unstably expressed gene in adipocytes.

The expression profiles of target genes can be markedly influenced depending on the choice of normalization genes. If the wrong reference gene is selected, it can result in false findings (23). This was reflected in our study. The expression profiles of 2 key adipogenic regulators, PPAR $\gamma 2$ and $\mathrm{C} / \mathrm{EBP} \alpha$, when normalized to $18 \mathrm{~S}$ and tubulin were similar to their raw expression levels (Fig. 4). PPAR $\gamma 2$ expression normalized to $18 \mathrm{~S}$ increased by 44- to 466-fold throughout 3T3-L1 adipocyte differentiation, exhibiting the well-established model of mature adipocytes. However, the increased folds of 2 target gene expression levels were markedly decreased when normalized to GAPDH and TFRC at various stages of adipocyte differentiation. The false results are expectedly drawn that the expression levels of 8 stable reference genes will markedly decrease if normalized to GAPDH.

This study clearly demonstrates the critical importance of reference gene validation for adipocyte differentiation studies, and highlights GAPDH and TFRC as unsuitable reference genes under this condition. 18S and HMBS are the most suitable reference genes for normalizing target genes during the course of 3T3-L1 adipocyte differentiation. The other 6 reference genes (actin, PIPA, Tubulin, 36-B4, HPRT and B2M) also showed relatively stable expression levels.

\section{Acknowledgements}

This study was funded by grants from the National Natural Science Foundation of China (30600294, 81070652, 81070617, 81170720, 81261120564 and 81270910).

\section{References}

1. Waki $\mathrm{H}$ and Tontonoz P: Endocrine functions of adipose tissue. Annu Rev Pathol 2: 31-56, 2007.

2. Kershaw EE and Flier JS: Adipose tissue as an endocrine organ. J Clin Endocrinol Metab 89: 2548-2556, 2004.

3. Gregoire FM, Smas CM and Sul HS: Understanding adipocyte differentiation. Physiol Rev 78: 783-809, 1998.

4. Pfaffl MW: The ongoing evolution of qPCR. Methods 50: 215-216, 2010.

5. Huggett J, Dheda K, Bustin S and Zumla A: Real-time RT-PCR normalisation; strategies and considerations. Genes Immun 6: 279-284, 2005.

6. Barroso I, Benito B, Garci-Jimenez C, Hernandez A, Obregon MJ and Santisteban P: Norepinephrine, tri-iodothyronine and insulin upregulate glyceraldehyde-3-phosphate dehydrogenase mRNA during Brown adipocyte differentiation. Eur J Endocrinol 141: 169-179, 1999.

7. Ferguson BS, Nam H, Hopkins RG and Morrison RF: Impact of reference gene selection for target gene normalization on experimental outcome using real-time qRT-PCR in adipocytes. PLoS One 5: e15208, 2010

8. Bas A, Forsberg G, Hammarström S and Hammarström ML: Utility of the housekeeping genes 18S rRNA, beta-actin and glyceraldehyde-3-phosphate-dehydrogenase for normalization in real-time quantitative reverse transcriptase-polymerase chain reaction analysis of gene expression in human $\mathrm{T}$ lymphocytes. Scand J Immunol 59: 566-573, 2004.

9. Stephens AS, Stephens SR and Morrison NA: Internal control genes for quantitative RT-PCR expression analysis in mouse osteoblasts, osteoclasts and macrophages. BMC Res Notes 4: 410, 2011

10. Vandesompele J, De Preter K, Pattyn F, Poppe B, Van Roy N, De Paepe A and Speleman F: Accurate normalization of real-time quantitative RT-PCR data by geometric averaging of multiple internal control genes. Genome Biol 3: RESEARCH0034, 2002.

11. Andersen CL, Jensen JL and Orntoft TF: Normalization of real-time quantitative reverse transcription-PCR data: a modelbased variance estimation approach to identify genes suited for normalization, applied to bladder and colon cancer data sets. Cancer Res 64: 5245-5250, 2004. 
12. Pfaffl MW, Tichopad A, Prgomet C and Neuvians TP: Determination of stable housekeeping genes, differentially regulated target genes and sample integrity: BestKeeper - Excel-based tool using pair-wise correlations. Biotechnol Lett 26: 509-515, 2004.

13. Derveaux S, Vandesompele J and Hellemans J: How to do successful gene expression analysis using real-time PCR. Methods 50:227-230, 2010.

14. Gorzelniak K, Janke J, Engeli S and Sharma AM: Validation of endogenous controls for gene expression studies in human adipocytes and preadipocytes. Horm Metab Res 33: 625-627, 2001.

15. Tatsumi K, Ohashi K, Taminishi S, Okano T, Yoshioka A and Shima M: Reference gene selection for real-time RT-PCR in regenerating mouse livers. Biochem Biophys Res Commun 374: 106-110, 2008

16. Stern-Straeter J, Bonaterra GA, Hormann K, Kinscherf R and Goessler UR: Identification of valid reference genes during the differentiation of human myoblasts. BMC Mol Biol 10: 66, 2009.

17. Farmer SR: Transcriptional control of adipocyte formation. Cell Metab 4: 263-273, 2006.

18. Zhou L, Wang X, Shao L, Yang Y, Shang W, Yuan G, Jiang B, Li F, Tang J, Jing $\mathrm{H}$ and Chen M: Berberine acutely inhibits insulin secretion from beta-cells through 3',5'-cyclic adenosine 5'-monophosphate signaling pathway. Endocrinology 149: 4510-4518, 2008.

19. Zhou L, Yang Y, Wang X, S. Liu S, Shang W, Yuan G, Li F, Tang J, Chen M and Chen J: Berberine stimulates glucose transport through a mechanism distinct from insulin. Metabolism 56: 405-412, 2007.

20. Zhou LB, Chen MD, Wang X, Song HD, Yang Y, Tang JF, Li FY, Xu MY and Chen JL: Effect of berberine on the differentiation of adipocyte. Zhonghua Yi Xue Za Zhi 83: 338-340, 2003 (In Chinese).

21. Huang C, Zhang Y, Gong Z, Sheng X, Li Z, Zhang W and Qin Y: Berberine inhibits 3T3-L1 adipocyte differentiation through the PPARgamma pathway, Biochem Biophys Res Commun 348: 571-578, 2006

22. Pham TP, Kwon J and Shin J: Berberine exerts anti-adipogenic activity through up-regulation of C/EBP inhibitors, CHOP and DEC2. Biochem Biophys Res Commun 413: 376-382, 2011.

23. Dheda K, Huggett JF, Bustin SA, Johnson MA, Rook G and Zumla A: Validation of housekeeping genes for normalizing RNA expression in real-time PCR. Biotechniques 37: 112-114, 116, 118-119, 2004.

24. Tricarico C, Pinzani P, Bianchi S, Paglierani M, Distante V, Pazzagli M, Bustin SA and Orlando C: Quantitative real-time reverse transcription polymerase chain reaction: normalization to rRNA or single housekeeping genes is inappropriate for human tissue biopsies. Anal Biochem 309: 293-300, 2002.

25. Touchberry CD, Wacker MJ, Richmond SR, Whitman SA and Godard MP: Age-related changes in relative expression of real-time PCR housekeeping genes in human skeletal muscle. J Biomol Tech 17: 157-162, 2006.

26. Poulos SP, Dodson MV and Hausman GJ: Cell line models for differentiation: preadipocytes and adipocytes. Exp Biol Med 235: 1185-1193, 2010.

27. Catalán V, Gómez-Ambrosi J, Rotellar F, Silva C, Rodríguez A, Salvador J, Gil MJ, Cienfuegos JA and Frühbeck G: Validation of endogenous control genes in human adipose tissue: relevance to obesity and obesity-associated type 2 diabetes mellitus. Horm Metab Res 39: 495-500, 2007.
28. Schmid H, Cohen CD, Henger A, Irrgang S, Schlondorff D and Kretzler M: Validation of endogenous controls for gene expression analysis in microdissected human renal biopsies. Kidney Int 64: 356-360, 2003.

29. Chechi K, Gelinas Y, Mathieu P, Deshaies Y and Richard D: Validation of reference genes for the relative quantification of gene expression in human epicardial adipose tissue. PLoS One 7: e32265, 2012.

30. Janke J, Engeli S, Gorzelniak K, Luft FC and Sharma AM: Resistin gene expression in human adipocytes is not related to insulin resistance. Obes Res 10: 1-5, 2002.

31. Choi K: The role of ghrelin and growth hormone secretagogues receptor on rat adipogenesis. Endocrinology 144: 754-759, 2003.

32. Liu YM, Lacorte JM, Viguerie N, Poitou C, Pelloux V, Guy-Grand B, Coussieu C, Langin D, Basdevant A and Clément K: Adiponectin gene expression in subcutaneous adipose tissue of obese women in response to shortterm very low calorie diet and refeeding. J Clin Endocrinol Metab 88: 5881-5886, 2003.

33. Sikand K, Singh J, Ebron JS and Shukla GC: Housekeeping gene selection advisory: glyceraldehyde-3-phosphate dehydrogenase (GAPDH) and beta-actin are targets of miR-644a. PLoS One 7: e47510, 2012

34. Li YL, Ye F, Hu Y, Lu WG and Xie X: Identification of suitable reference genes for gene expression studies of human serous ovarian cancer by real-time polymerase chain reaction. Anal Biochem 394: 110-116, 2009.

35. Rolland V, Dugail I, Le Liepvre X and Lavau M: Evidence of increased glyceraldehyde-3-phosphate dehydrogenase and fatty acid synthetase promoter activities in transiently transfected adipocytes from genetically obese rats. J Biol Chem 270: 1102-1106, 1995

36. Dugail I, Quignard-Boulangé A, Le Liepvre X, Ardouin B and Lavau M: Gene expression of lipid storage-related enzymes in adipose tissue of the genetically obese Zucker rat. Co-ordinated increase in transcriptional activity and potentiation by hyperinsulinaemia. Biochem J 281: 607-611, 1992.

37. Alexander MC, Lomanto M, Nasrin N and Ramadka C: Insulin stimulates glyceraldehyde-3-phosphate dehydrogenase gene expression through cis-acting DNA sequences. Proc Natl Acad Sci USA 85: 5092-5096, 1988.

38. Wang ZX, Jiang CS, Liu L, Wang XH, Jin HJ, Wu Q and Chen Q: The role of Akt on arsenic trioxide suppression of 3T3-L1 preadipocyte differentiation. Cell Res 15: 379-386, 2005.

39. Gary-Bobo M, Elachouri G, Scatton B, Le Fur G, Oury-Donat F and Bensaid $\mathrm{M}$ : The cannabinoid $\mathrm{CB} 1$ receptor antagonist rimonabant (SR141716) inhibits cell proliferation and increases markers of adipocyte maturation in cultured mouse 3T3 F442A preadipocytes. Mol Pharmacol 69: 471-478, 2006.

40. Tanner LI and Lienhard GE: Insulin elicits a redistribution of transferrin receptors in 3T3-L1 adipocytes through an increase in the rate constant for receptor externalization. J Biol Chem 262: 8975-8980, 1987.

41. Gabrielsen JS, Gao Y, Simcox JA, Huang J, Thorup D, Jones D, Cooksey RC, Gabrielsen D, Adams TD, Hunt SC, Hopkins PN, Cefalu WT and McClain DA: Adipocyte iron regulates adiponectin and insulin sensitivity. J Clin Invest 122: 3529-3540, 2012. 